\title{
Changes before and after COVID-19 pandemic on the personal hygiene behaviors and incidence of peritonitis in peritoneal-dialysis patients: a multi-center retrospective study
}

\author{
Yanglin $\mathrm{Hu}^{1} \odot \cdot \mathrm{Li} \mathrm{Xu}^{2} \cdot$ XiaoHui Wang $^{3} \cdot$ Xiaofei Qin $^{4} \cdot$ Sheng Wan ${ }^{1} \cdot$ Qing Luo ${ }^{1}$. Yanqiong Ding ${ }^{1} \cdot$ Xiaofen Xiao $^{3}$. \\ Fei Xiong ${ }^{1}$
}

Received: 4 January 2021 / Accepted: 6 June 2021 / Published online: 19 June 2021

(c) The Author(s), under exclusive licence to Springer Nature B.V. 2021

\begin{abstract}
Background The impact of Coronavirus disease (COVID-19) pandemic and its influence on personal hygiene behaviors and peritonitis rate in peritoneal-dialysis patients is unknown.

Methods A multi-center retrospective study was conducted. We reviewed all the cases of peritoneal-dialysis (PD) patients from four major PD centers in Wuhan before and after COVID-19. There were 567 patients enrolled in total. Information was collected on personal hygiene behaviors, basic clinical characteristics, lab results, peritonitis details. We used Chisquare analysis to compare the personal hygiene behaviors, and used Chi-square goodness-of-fit analysis to compare the peritonitis rates before and after COVID-19. Multivariate logistic regression analysis was used to analyze the risk factors for peritonitis rate.

Results There were no significant differences on peritonitis rates in six-month period before and after COVID-19 ( $p=0.0756$, Fig. 2 and Table 3). But Gram-positive infections decreased dramatically $(p=0.0041$, Table 4). Personal hygiene behaviors such as length of time for washing hands when performing PD treatment, the frequency of washing hands before PD treatment and six general behaviors had significant differences $(P<0.05$ Table 2$)$. The multivariate logistic regression analysis showed never washing hands before PD treatment and serum albumin level were the risk factors of peritonitis during COVID-19 (OR 14.408, 95\% CI 3.930-52.821, $P=0.0002$; OR 4.681, 95\% CI 1.755-12.485, $P=0.002$, Table 5).

Conclusions The COVID-19 pandemic had a significant positive influence on personal hygiene behaviors. Peritonitis rate did not significantly decrease but Gram-positive infections dramatically decreased. Never hand washing before PD treatment and serum albumin were the risk factors for peritonitis. We should emphasize hand washing before PD treatment in training and re-training program.
\end{abstract}

Keywords Peritoneal dialysis $\cdot$ Peritonitis $\cdot$ COVID-19 $\cdot$ Hand hygiene $\cdot$ Risk factor

Yanglin $\mathrm{Hu}$ and $\mathrm{Li} \mathrm{Xu}$ contributed equally to this paper

Fei Xiong

xiongf23@sina.com

1 Department of Nephrology, Wuhan No. 1 Hospital, Zhongshan Road 215\# Qiaokou Area, Wuhan 430022, Hubei, China

2 Department of Nephrology, The Central Hospital of Wuhan, Tongji Medical College, Huazhong University of Science and Technology, Wuhan, China

3 Department of Nephrology, Wuhan No. 5 Hospital, Wuhan, China

4 Department of Nephrology, The People's Hospital of Huangpi, Wuhan, China

\section{Introduction}

During the Coronavirus disease (COVID-19) pandemic, the management of peritoneal-dialysis (PD) patients and PD dialysis treatment for acute kidney injury are commonly discussed [1-3]. But the change in peritonitis rate and the change in personal hygiene behaviors are seldom discussed.

As is known, peritonitis is a common and serious complication of PD and can lead to PD failure and even death. Both patients related factors and therapy related factors are crucial to reduce the peritonitis rate. However, when it comes to real life, few patients do exactly as they were trained [4, 5].

The COVID-19 pandemic brought a dramatic change towards people's personal life. China built extremely strict 
rules for the whole country, especially in Wuhan at the very beginning. The city was locked down, no public traffic, no restaurants were open. All the people stayed at home. Everyone wear masks and people were more vigilant and aware of hand hygiene.

Since the COVID-19 pandemic changed environmental and personal behaviors, we are curious about the role of that change for PD patients. And also, we are interested in which specific personal behavior influenced peritonitis rate the most. Till now, little is known about that. Our study will be the first to investigate the changes before and after COVID-19 pandemic on personal hygiene behaviors and the rate of peritonitis in peritoneal-dialysis patients. It is also the first study about the risk factors of peritonitis during COVID-19 pandemic.

\section{Materials and methods}

\section{Study design and participants}

We reviewed all the clinical and personal information of prevalent PD patients in the 4 major PD centers in Wuhan before and after COVID-19 pandemic including those with and without peritonitis (Fig. 1).

Since the breakout of COVID-19 pandemic was in December 2019. We defined the time after COVID-19 as the period between January 1st, 2020 and June 30th, 2020. Accordingly, we defined the time before COVID-19 as the same period last year, to exclude the seasonal climate influence which was between Jan 1st 2019 to June 30th 2019 [6].

The inclusion criteria of the study were: (a) routine follow-up defined as at least once every six months, (b) $>18$ years of age, (c) consent to participate in the research. (d) visiting the centers in both periods including before and after COVID- 19 .

The exclusion criteria of the study were: (a) patients who had psychological-cognitive impairment and psychiatric problems were excluded (defined as any neurological or psychiatric disorders that lead to the inability to perform PD by him/herself or inability to respond to questionnaire); (b) combined HD and PD therapy were excluded; (c) patients who had PD treatment for AKI were excluded.

Baseline data from the participants were collected, these include patient demographics (age, sex, education), cause of end-stage renal disease (ESRD), body mass index (BMI), comorbidities (Diabetes mellitus, hypertension, cardiovascular disease, cerebrovascular disease), PD modality (continuous ambulatory PD [CAPD], day time ambulatory PD [DAPD] and automated PD [APD]), dialysis efficacy and peritoneal membrane transport quality and laboratory results.
In June 2020, we did a thorough investigation on all patients about the personal hygiene behaviors before and after COVID-19. All patients were given an anonymous Questionnaire Form (electronic or paper) on personal hygiene behavior self-efficacy scale. The questions were mainly about hand washing and mask-wearing behaviors before and after COVID-19. There were in total 11 personal hygiene behaviors including: the frequency of washing hands before PD treatment, the frequency of washing hands just before connecting transfer set, the frequency of washing hands before disconnecting the transfer set, the length of time for washing hands, the frequency of hand washing when not performing PD treatment, the habit of washing hand after defecation, the condition of mask-wearing in the environment where PD is applied, the frequency of changing a new mask, the frequency of going out, of dining out, of cleaning the living environment(see supplements). The standard hand-washing protocol adopted was the Center for Disease Control (CDC) protocol [7-9]. And all the patients were trained according to the protocol when PD treatment started $[7,8]$.

All episodes of peritonitis in PD patients in our unit before and after COVID-19 were also reviewed. The diagnosis of peritonitis was based on at least two of the following: (1) clinical features consistent with peritonitis, i.e. abdominal pain and/or cloudy dialysis effluent; (2) dialysis effluent white cell count $>100 / \mu \mathrm{L}$ or $>0.1 \times 109 / \mathrm{L}$ (after a dwell time of at least $2 \mathrm{~h}$ ), with $>50 \%$ polymorphonuclear; and (3) positive dialysis effluent culture [8]. The time of onset, the suspected etiology and microbiology result for peritonitis were examined. PD fluid sample was collected when peritonitis was clinically suspected. Sampling was done by dialysis nurses and every step was performed under the guidance of ISPD.

The peritonitis rate should be reported as the number of episodes per patient-year as recommended [10]. To find out what was the trend of peritonitis rate after COVID-19 pandemic, we compared the peritonitis rate before and after COVID-19 breakout by drawing a curve depicting the peritonitis rate of each month in the periods before and after COVID-19. Each month's peritonitis rate was calculated using the conventional cohort-specific peritonitis rate methods based yearly basis. For example, peritonitis rate of January 2019 was calculated as the peritonitis rate between January 2018 and January 2019. To calculate the peritonitis rate of each month, we further retrieved the number of peritonitis episodes from January 2018 to June 2020.

\section{Statistic analysis}

Quantitative variables were expressed as mean (standard deviation), or median (inter quartile range) depending on whether they fitted the normal distribution. For qualitative 


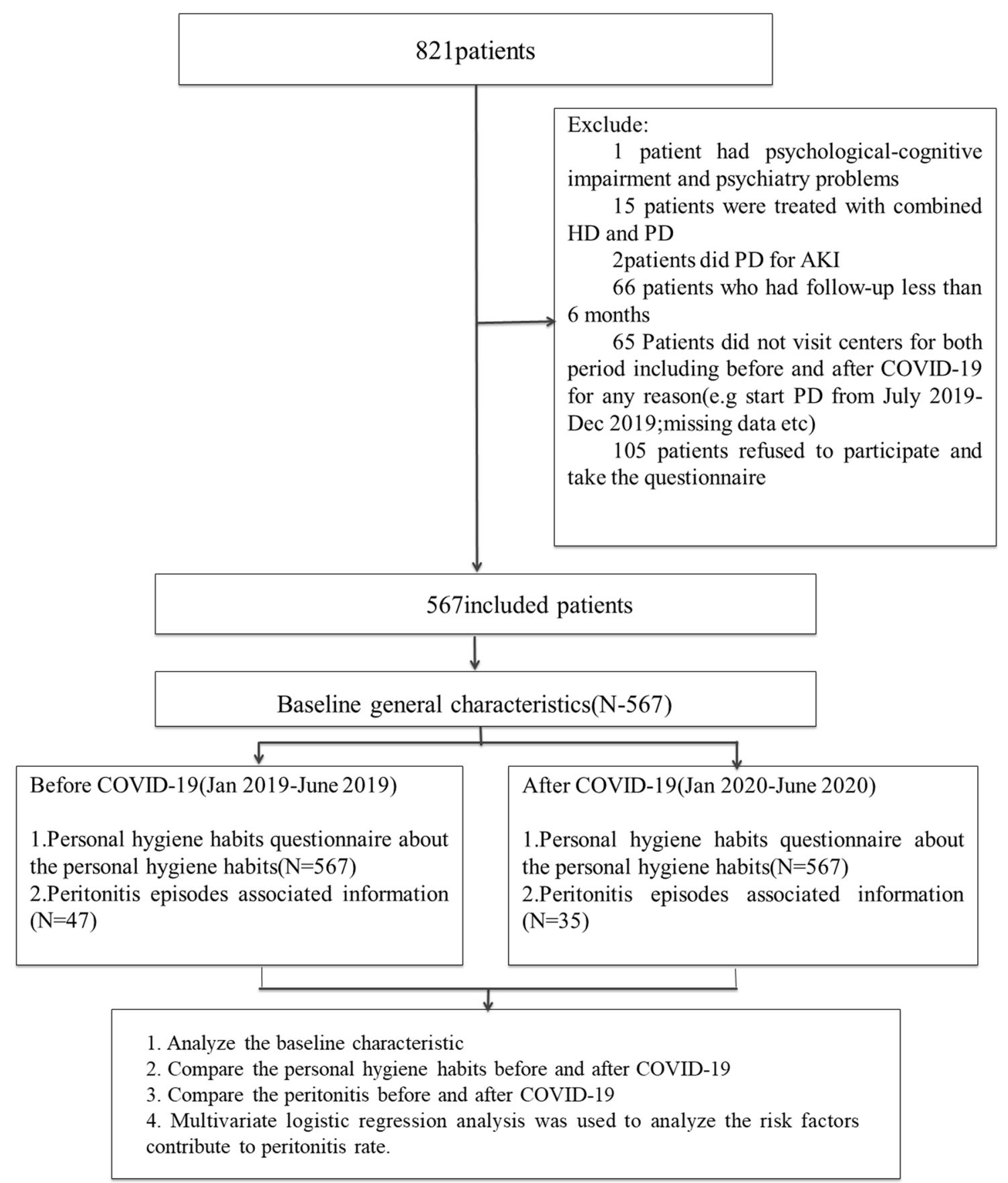

Fig. 1 Patient disposition

variables, statistical description was expressed as frequency (percentage). Chi-Square analysis was used to compare the personal hygiene behaviors before and after COVID-19. Chisquare goodness-of-fit analysis was used to compare peritonitis rate between the time before and after COVID-19. ChiSquare analysis and Fisher's exact test were used to compare the suspicious etiology and microbiology before and after COVID-19. Multivariate logistic regression analysis was used to analyze the risk factors contributed to peritonitis rate. The potential covariates were chosen by professional judgment. Variables with a $p$ value $<0.10$ in the univariate analysis were included in the multivariate logistic regression analysis. The comparative risk of an incidence rate exposed with covariate was then stated as an odds ratio (OR) [95\% confidence interval (CI)]. Statistical software was Statistical software was SAS 9.4, and all hypothesis tests were twosided tests with a significance level of 0.05 . 


\section{Results}

\section{General characteristics}

In total, $567 \mathrm{PD}$ patients in 4 peritoneal-dialysis centers in Wuhan were enrolled in the study. The basic characteristics of all those patients were analyzed and summarized in Table 1.The mean age of the patients was $57.42 \pm 13.25$ years old, the mean BMI was $22.47 \pm 3.93 \mathrm{~kg} /$ $\mathrm{m} 2,49.6 \%$ were male, $40.48 \%$ had high-school education or above. The most common primary disease was primary glomerulonephritis(42.11\%). The most common co-morbidity was hypertension(31.1\%). The most common PD pattern was CAPD(93.9\%). The mean total $\mathrm{KT} / \mathrm{V}$ was $1.86 \pm 0.5 \mathrm{~mL} /$ min. The median D/P creatinine was 0.63 (IQR 0.55-0.71) (Table 1).

Table 1 Baseline general characteristics

\begin{tabular}{|c|c|}
\hline \multicolumn{2}{|l|}{ Characteristics } \\
\hline Age (years), mean (SD) & $57.42(13.25)$ \\
\hline \multicolumn{2}{|l|}{ Gender male, $n(\%)$} \\
\hline Male & $281(49.56)$ \\
\hline Female & $286(50.44)$ \\
\hline BMI $\left(\mathrm{kg} / \mathrm{m}^{2}\right)$, mean $(\mathrm{SD})$ & $22.47(3.93)$ \\
\hline \multicolumn{2}{|l|}{ Level of education, $n(\%)$} \\
\hline Primary school or less & $176(31.04)$ \\
\hline Secondary school & $162(28.57)$ \\
\hline High school and above & $229(40.39)$ \\
\hline Total KT/Vmean (SD) & $1.86(0.5)$ \\
\hline D/P creatinine (median, IQR) & $0.63(0.55-0.71)$ \\
\hline \multicolumn{2}{|l|}{ Modality of PD, $n(\%)$} \\
\hline CAPD & $532(93.83)$ \\
\hline DAPD & $17(3.00)$ \\
\hline APD & $3(0.53)$ \\
\hline Others & $15(2.65)$ \\
\hline \multicolumn{2}{|l|}{ Primary disease of ESRD, $n(\%)$} \\
\hline Diabetes & $74(13.05)$ \\
\hline Hypertension & $169(29.81)$ \\
\hline Glomerulonephritis & $238(41.96)$ \\
\hline Lupus & $12(2.11)$ \\
\hline Polycystic kidney & $9(1.59)$ \\
\hline Other & $65(11.46)$ \\
\hline \multicolumn{2}{|l|}{ Comorbidity of ESRD, $n(\%)$} \\
\hline Diabetes mellitus & $43(7.58)$ \\
\hline Hypertension & $176(31.04)$ \\
\hline Cardiovascular disease & $127(22.40)$ \\
\hline Cerebrovascular disease & $24(4.23)$ \\
\hline $\mathrm{HBV}$ & $3(0.53)$ \\
\hline Tumor & $3(0.53)$ \\
\hline
\end{tabular}

\section{Differences of personal hygiene behaviors before and after COVID-19}

There were in total 11 personal hygiene behaviors studied, with the results showing 8 of them showing significant differences. According to our analysis, the length of time for washing hands, the frequency of washing hands before PD treatment and when not performing PD treatment, the condition of mask-wearing in the environment where PD is applied, the frequency of changing a new mask, the frequency of going out, of dining out, of cleaning the living environment had significant differences before and after COVID-19 $(P<0.05$ Table 2$)$. And the percentage of patients who had better personal hygiene behaviors increased dramatically which showed the positive influence of COVID-19 breakout on patients' personal hygiene behaviors $(P<0.05$ Table 2$)$. The frequency of washing hands when connecting and disconnecting the transfer set, and the habit of washing hand after defecation did not have significant differences before and after COVID-19 $(p>0.05)$.

\section{Difference of peritonitis rates and gram positive infection in six-month period before and after COVID-19}

After comparing the peritonitis rate in six-month period before and after COVID-19, there were no significant difference $(P=0.0756)$. But the curve showed a decreasing trend in peritonitis rate after COVID-19 (Fig. 2 and Table 3). The suspecting etiology and microbiologic results of the peritonitis episodes before and after COVID-19 were summarized in Table 4 showing that the most common bacteria were Gram positive and the most common etiology was improper hand hygiene (Table 4). The gram-positive infection rate significantly decreased from 76.6 to $45.71 \%$ after COVID-19 breakout ( $p=0.0041$, Table 4$)$.

\section{Risk factors for peritonitis}

48 clinical factors, determined at the time of diagnosis, were analyzed for their possible role as indicators of risk factors for peritonitis. The 48 clinical factors include: the hand hygiene habits, such as the frequency of washing hands before PD treatment before and after COVID-19 (never, occasionally, frequently, always); the frequency of washing hands just before the connecting transfer set(never, occasionally, frequently, always) etc.; and the basic characteristics such as gender, age, BMI, total time of PD etc.; and lab results such as PET, Total KT/V, albumin etc. (see supplements). Risk factors for peritonitis after a univariate analysis are listed in Table 5. When adjusted in the regression model, only never washing hands before PD treatment and albumin 
Table 2 Comparison of personal hygiene behaviors before and after COVID-19

\begin{tabular}{lcccc}
\hline Personal hygiene behavior & $\begin{array}{l}\text { Before } \\
\text { COVID-19 } N \\
(\%)\end{array}$ & After COVID-19N(\%) & Chi-Square & $P$ value \\
\hline The frequency of washing hands before PD treatment & & \\
Never & $9(1.59)$ & $6(1.06)$ & 30.58 & \\
Occasionally & $89(15.70)$ & $55(9.70)$ & \\
Frequently & $226(39.86)$ & $163(28.75)$ & \\
Always & $243(42.86)$ & $343(60.49)$ & 0.001 \\
The frequency of washing hands just before connecting transfer set & \\
Never & $20(3.53)$ & $15(2.65)$ & \\
Occasionally & $78(13.76)$ & $46(8.11)$ & \\
Frequently & $168(29.63)$ & $246(43.39)$ & \\
Always & $301(53.09)$ & $260(45.86)$ & \\
The frequency of washing hands before disconnecting transfer set & \\
Never & $43(7.58)$ & $39(6.88)$ & \\
Occasionally & $90(15.87)$ & $98(17.28)$ & \\
Frequently & $206(36.33)$ & $251(44.27)$ & \\
Always & $228(40.21)$ & $179(31.57)$ &
\end{tabular}

The length of time for washing hands in accordance with the hygienic hand-washing technique of at least $15 \mathrm{~s}$

$\begin{array}{lll}\text { Never } & 53(9.35) & 17(3.00) \\ \text { Occasionally } & 144(25.40) & 136(23.99) \\ \text { Frequently } & 139(24.51) & 149(26.28) \\ \text { Always } & 231(40.74) & 265(46.74)\end{array}$

The frequency of hand washing when not performing the procedure

\begin{tabular}{lclll} 
Never & $12(2.12)$ & $6(1.06)$ & 5.55 & 0.0185 \\
Occasionally & $206(36.33)$ & $143(25.22)$ & & \\
Frequently & $219(38.62)$ & $303(53.44)$ & & \\
Always & $130(22.93)$ & $115(20.28)$ & & \\
\multicolumn{2}{l}{ The habit of washing hand after defecation } & & 0.6161 \\
Yes & $20(3.53)$ & $17(3.00)$ &
\end{tabular}

The condition of mask-wearing in the environment where PD is applied

\begin{tabular}{|c|c|c|c|c|}
\hline Never & $155(27.34)$ & $104(18.34)$ & 44.49 & $<0.0001$ \\
\hline Occasionally & $256(45.15)$ & $194(34.22)$ & & \\
\hline Frequently & $91(16.05)$ & $142(25.04)$ & & \\
\hline Always & $65(11.46)$ & $127(22.40)$ & & \\
\hline \multicolumn{5}{|c|}{ The frequency of changing a new mask } \\
\hline Occasionally & $278(49.03)$ & $93(16.40)$ & 202.91 & $<0.0001$ \\
\hline Sometimes & $201(35.45)$ & $163(28.75)$ & & \\
\hline Frequently & $57(10.05)$ & $201(35.45)$ & & \\
\hline Always & $31(5.47)$ & $110(19.40)$ & & \\
\hline \multicolumn{5}{|c|}{ The frequency of going out } \\
\hline Never & $89(15.70)$ & $185(32.63)$ & 68.62 & $<0.0001$ \\
\hline Occasionally & $246(43.39)$ & $277(48.85)$ & & \\
\hline Frequently & $186(32.80)$ & $74(13.05)$ & & \\
\hline Always & $46(8.11)$ & $31(5.47)$ & & \\
\hline \multicolumn{5}{|c|}{ The frequency of dining out } \\
\hline Never & $199(35.10)$ & $419(73.90)$ & Fisher 's exact & $<0.0001$ \\
\hline Occasionally & $303(53.44)$ & $121(21.34)$ & & \\
\hline Frequently & $61(10.76)$ & $24(4.23)$ & & \\
\hline Always & $4(0.71)$ & $3(0.53)$ & & \\
\hline
\end{tabular}


Table 2 (continued)

\begin{tabular}{lllll}
\hline Personal hygiene behavior & $\begin{array}{l}\text { Before } \\
\text { COVID-19N }\end{array}$ & After COVID-19N(\%) & Chi-Square & $P$ value \\
& $(\%)$ & & \\
\hline The frequency of cleaning the living environment & & \\
Never & $17(3.00)$ & $8(1.41)$ & 44.77 & \\
Occasionally & $101(17.81)$ & $56(9.88)$ & \\
Frequently & $334(58.91)$ & $291(51.32)$ & \\
Always & $115(20.28)$ & $212(37.39)$ & \\
\hline
\end{tabular}

remained an independent predictor risk factor of peritonitis before and after COVID-19 (OR 14.408, 95\% CI: $3.930-52.821, P=0.0002$; OR $4.681,95 \% \mathrm{CI}$ : $1.755-12.485, P=0.0020$, Table 5).

\section{Discussion}

In the present study, we revealed changes to peritonitisrelated issues after the COVID-19 breakout: personal hygiene behaviors significantly improved, and peritonitis

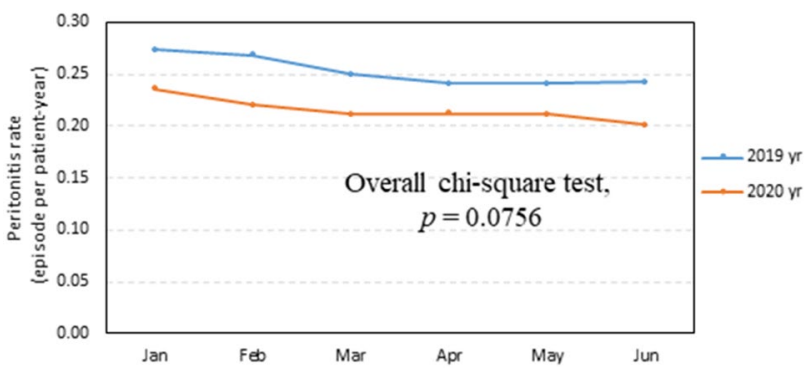

Fig. 2 Peritonitis rate in January to June 2020, which is during the time after COVID-19 pandemic compare to last year

Table 3 Peritonitis in January to June 2020, which is during the time after COVID-19 pandemic compare to last year

\begin{tabular}{cllll}
\hline Year & Month & Patient-years & Case number & Peritonitis rate \\
\hline 2019 & Jan & 574 & 157 & 0.2735 \\
& Feb & 577 & 155 & 0.2686 \\
& Mar & 581 & 145 & 0.2500 \\
& Apr & 583 & 141 & 0.2410 \\
& May & 586 & 141 & 0.2406 \\
& Jun & 589 & 143 & 0.2428 \\
2020 & Jan & 611 & 144 & 0.2357 \\
& Feb & 614 & 135 & 0.2199 \\
& Mar & 616 & 130 & 0.2110 \\
& Apr & 618 & 131 & 0.2120 \\
& May & 621 & 131 & 0.2110 \\
& Jun & 623 & 125 & 0.2006 \\
\hline
\end{tabular}

*Peritonitis rate: episode per patient-year rate did not have significant statistic difference in sixmonth period after COVID-19 breakout. However, the rate of Gram-positive infection significantly decreased. The risk factors that contributed to peritonitis during the COVID-19 pandemic were: never washing hands before PD treatment and serum albumin level. This is the first and largest multicenter retrospective study to investigate the change of peritonitis-related issues before and after COVID-19. Therefore, our study presents new data for the literature.

Hand hygiene is considered to be the most cost-effective and efficacious method for preventing contamination-related PD infection. Noncompliance with hand hygiene is very common and is highly associated with higher peritonitis rate [11-13]. An Italian study found that, after a mean of

Table 4 Suspicious etiology and microbiologic causes of the peritonitis episodes before and after COVID-19

\begin{tabular}{|c|c|c|c|}
\hline & $\begin{array}{l}\text { Before } \\
\text { COVID- } \\
19 N(\%)\end{array}$ & $\begin{array}{l}\text { After } \\
\text { COVID- } \\
19 N(\%)\end{array}$ & $P$ value \\
\hline \multicolumn{4}{|l|}{ Suspicious etiology } \\
\hline Diet & $11(23.40)$ & $8(22.86)$ & 0.488 \\
\hline Improper hand hygiene & $14(29.79)$ & $16(45.71)$ & 0.7117 \\
\hline Others & $22(46.81)$ & $11(31.43)$ & 0.2344 \\
\hline \multicolumn{4}{|l|}{ Microbiologic causes } \\
\hline Gram positive & $36(76.6)$ & $16(45.71)$ & 0.0041 \\
\hline Staphylococcus aureus & $5(10.64)$ & $3(8.57)$ & $0.6894 *$ \\
\hline $\begin{array}{l}\text { Coagulase negative staphy- } \\
\text { lococci }\end{array}$ & $9(19.15)$ & $2(5.71)$ & $0.4679 *$ \\
\hline Streptococcus pneumoniae & $3(6.38)$ & $0(0.00)$ & $0.5439 *$ \\
\hline Enterococcus & $4(8.51)$ & $3(8.57)$ & $0.6619 *$ \\
\hline Other gram-positive bacteria & $15(31.91)$ & $8(22.86)$ & 0.5766 \\
\hline Gram-negative & $7(14.89)$ & $6(17.14)$ & $0.7827 *$ \\
\hline Escherichia coli & $5(10.64)$ & $2(5.71)$ & $0.2861^{*}$ \\
\hline Klebsiella pneumoniae & $0(0.00)$ & $0(0.00)$ & - \\
\hline Pseudomonas aeruginosa & $1(2.13)$ & $2(5.71)$ & $0.5594^{*}$ \\
\hline Other gram-negative bacteria & $1(2.13)$ & $2(5.71)$ & $0.5594 *$ \\
\hline Fungus & $0(0.00)$ & $1(2.86)$ & $0.4268^{*}$ \\
\hline Mycobacterial species & $0(0.00)$ & $0(0.00)$ & - \\
\hline No Growth & $4(8.51)$ & $12(34.29)$ & 0.0036 \\
\hline
\end{tabular}

*Fisher's exact test 
Table 5 Logistic regression analysis of risk factors for peritonitis

\begin{tabular}{|c|c|c|c|c|c|c|}
\hline & $\begin{array}{l}\text { Univariate analysis OR }(95 \% \\
\mathrm{CI})\end{array}$ & Wald ChiSq & $P$ value & $\begin{array}{l}\text { Multivariate analysis OR } \\
(95 \% \mathrm{CI})\end{array}$ & Wald ChiSq & $P$ value \\
\hline $\begin{array}{l}\text { Never wash hands before PD } \\
\text { treatment }\end{array}$ & $10.770(3.433-33.786)$ & 13.4284 & 0.0002 & $14.408(3.930-52.821)$ & 13.5180 & 0.0002 \\
\hline $\begin{array}{l}\text { Never wash hands just before } \\
\text { connecting the transfer set }\end{array}$ & $5.848(2.313-14.784)$ & 9.6063 & 0.0019 & & & \\
\hline $\begin{array}{l}\text { Time of washing hands in } \\
\text { accordance with the hygienic } \\
\text { hand-washing technique } \\
\text { never reach } 15 \mathrm{~s}\end{array}$ & $3.179(1.400-7.218)$ & 6.3859 & 0.0115 & & & \\
\hline $\begin{array}{l}\text { Never wash hand when not } \\
\text { performing the procedure }\end{array}$ & $5.245(1.284-21.417)$ & 4.0747 & 0.0435 & & & \\
\hline $\begin{array}{l}\text { Seldom clean the living envi- } \\
\text { ronment }\end{array}$ & $8.092(2.932-22.333)$ & 17.7372 & $<0.0001$ & & & \\
\hline Abnormal Albumin & $4.217(1.677-10.605)$ & 9.3577 & 0.0022 & $4.681(1.755-12.485)$ & 9.5074 & 0.0020 \\
\hline
\end{tabular}

33 months on PD, $23 \%$ did not follow the correct exchange procedures aiming at preventing infections: $9 \%$ did not use a mask, $6 \%$ did not wash hands, and $8 \%$ did not pay enough attention to general hygiene concepts during exchange procedures. And collectively those $23 \%$ patients had higher peritonitis rate [12]. The latest International Society for Peritoneal Dialysis (ISPD) guidelines suggests hand hygiene should be emphasized during patient training [8, 13]. And the suggested efficient methods of washing hands by ISPD goes along with the CDC recommendations which is $70 \%$ alcohol-based hand rubs and antimicrobial soap for at least $15 \mathrm{~s}[8,9,13]$. Wearing a mask is optional in ISPD guideline [8]. Although the guideline gives us general recommendations on hand hygiene and mask-wearing, the number of studies focusing on hand washing, mask wearing and other specific personal hygiene habits to prevent PD-related infections are limited. The critical elements of a training program that determine the peritonitis rate remain undefined [10]. Many PD interventions and practices including duration of hand washing, type of hand-washing agent used, still require formal evaluation by clinical studies [14-16].

Our study showed two protocol-required personal hygiene behaviors that improved after COVID-19 breakout $(p<0.05$, Table 2). The first was the frequency of washing hands before PD treatment, which should be done following 6-step hand-washing technique. The other was the length of time for washing hand which should be at least $15 \mathrm{~s}$. Besides that, 6 general personal hygiene behaviors also improved among PD patients, including hand washing while not performing PD, mask-wearing during PD treatment, the change of masks, frequency of dining out and going out, the frequency of cleaning the living environment. The results showed a complete thorough change of personal hygiene behaviors (In China, dining out and going out is not considered to be good personal hygiene behaviors because eating and staying at home is considered to be cleaner, safer and healthier). In other words, patients were more compliant to protocols and were cleaner in general. Since noncompliance with exchange protocols, especially noncompliance with hand hygiene, is very common and is highly associated with higher rate of peritonitis [11-13]. The improved required behaviors also meant increased compliance rate after COVID-19 which may lead to decrease in peritonitis rate, especially the Grampositive infections.

We further analyzed the peritonitis conditions in our study. Like other facilities in the world, Gram-positive organisms were the leading cause of PD-related peritonitis [10, 17-19]. When comparing the peritonitis rate before and after COVID-19 breakout, there was a decreasing trend in year 2020 compared with year 2019. But there was not statistical significance ( $p=0.0756$, Fig. 2 and Table 3 ). But when we further studied the microbiology distribution, the Grampositive infection rate significantly decreased from $76.6 \%$ to $45.71 \%$ after COVID-19 breakout (Table 4 ). The results show that better personal hygiene behaviors, influenced by COVID-19 breakout, can decrease the Gram-positive infection. But although personal hygiene behaviors changed dramatically, to result in significant decrease peritonitis rate, more methods and effort or longer time should be needed.

When we evaluated the risk factors for peritonitis, we found that never washing hands before PD treatment increased the risk of peritonitis by 14.4 -fold ( $p=0.0002$, Table 5) during COVID-19 pandemic. As we discussed above, hand hygiene was an important prevention practices against peritonitis. Among the 11 personal hygiene behaviors we included in the logistic multivariate analysis, this was the only one turning out to be a risk factor for peritonitis during COVID-19. Although the percentage of people who never wash hand before treatment decreased from 1.59 to $1.06 \%$ (Table 2), never washing hand before treatment still remained the risk factor during COVID-19 pandemic. From that result, we can conclude that, by further emphasizing the 
hand hygiene habit of washing hands before PD treatment after COVID-19 pandemic can decrease the rate of peritonitis dramatically. And that conclusion can influence on PD treatment training and re-training program as well. Serum albumin level was found to be another risk factor for peritonitis during COVID-19 in our multivariate model: it increased the peritonitis risk 4.68-fold ( $p=0.002$, Table 5) during COVID19 pandemic. It is understandable because the abnormality of serum albumin levels, most of the time hypoalbuminemia, is a marker of malnutrition, protein loss, and altered inflammatory response and other studies in the literature and guidelines also have the same finding $[8,14,20]$.

Our study has several potential limitations: first, the change of personal hygiene behaviors was based on questionnaire which was relatively objective. And because the questionnaire was taken in June 2020, it may have recall bias about the data before COVID-19 pandemic. But the questionnaire was done through several ways and advanced nurse specialists helped with all the questionnaire by explaining every question we listed. And also, we gave patients enough time to fill out the questionnaire to reduce the recall bias as much as possible; Second, it is a retrospective observational study instead of a prospective interventional study. It cannot give a high evidence support of causative conclusion. Better solid evidence could be made if a prospective interventional study could be designed and conducted; Third, the observation length for the change is relatively short, longer observation could give more solid conclusions. We intend to extend our observation to a year or more to see the changes after COVID-19 breakout especially the trend of peritonitis rate in longer time.

In conclusion, the COVID-19 pandemic breakout led to an improvement of personal hygiene behaviors. Peritonitis rates did not differ significantly after COVID-19 but Grampositive infection dramatically decreased. Never washing hands before PD treatment and serum albumin were the risk factors for peritonitis during COVID-19 pandemic. Our results showed that COVID-19 pandemic improved personal hygiene behaviors, can decrease the Gram-positive infection rate which provide a bright side of the current stressful situation. However, more effort should be done to improve the peritonitis rate especially by encouraging hand washing before PD treatment. Till the time of writing this paper, the COVID-19 pandemic lasted just more than a year. Further studies with longer observation periods are needed to study the influence of COVID-19 on peritonitis rate.

Acknowledgements All of the 4 dialysis centers involved are appreciated. We also gratefully acknowledge the dedication of the nursing and medical staff members of all the participating centers and the generosity of the study participants or family members, without whom this study could not have been completed. We also thank Dr.Zhen Fu and Dr Yanran Duan for their advices in data analysis and support. We thank Dr.Fatima Al-Hashimi in revising the language used in this paper.
Authors' contributions FX and YH made a substantial contribution to the concept or design of the work. YH made a substantial contribution to analysis and interpretation of data, drafted the article and revised it critically for important intellectual content. LX revised the paper and contribute the data of the article. XHW, XQ, SW, Q1, YD, XX collected the original data. Each author should have participated sufficiently in the work to take public responsibility for appropriate portions of the content.

\section{Declarations}

Conflict of interest None of the authors have any conflict of interest to disclose. We confirm that we have read the Journal's position on issues involved in ethical publication and affirm that this report is consistent with those guidelines.

Ethical approval The institutional ethics board of Wuhan No. 1 Hospital approved this study. We conducted direct communication with patients or their families as much as possible. Written informed consent was waived because of the rapid emergence of this infectious disease, and verbal consent obtained.

\section{References}

1. El Shamy O, Patel N, Abdelbaset MH, Chenet L, Tokita J, Lookstein R, Lee DS, Cohen NA, Sharma S, Uribarri J (2020) Acute start peritoneal dialysis during the COVID-19 pandemic: outcomes and experiences. JASN. https://doi.org/10.1681/ASN. 2020050599

2. Sourial MY, Sourial MH, Dalsan R, Graham J, Ross M, Chen W, Golestaneh L (2020) Urgent peritoneal dialysis in patients with COVID-19 and acute kidney injury: a single- center experience in a time of crisis in the United States. Am J Kidney Dis. https:// doi.org/10.1053/j.ajkd.2020.06.001

3. Brown EA, Perl J (2020) Increasing peritoneal dialysis use in response to the COVID-19 pandemic: will it go viral? JASN. https://doi.org/10.1681/ASN.2020050729

4. Figueiredo AE, Bernardini J, Bowes E, Hiramatsu M, Price V, Su C, Walker R, Brunier G (2016) A syllabus for teaching peritoneal dialysis to patients and caregivers. Perit Dial Int 36:592-605

5. Zhang L, Hawley CM, Johnson DW (2016) Focus on peritoneal dialysis training: working to decrease peritonitis rates. Nephrol Dial Transplant 31:214-222

6. Szeto CC, Chow KM, Wong TY, Leung CB, Li PK (2003) Influence of climate on the incidence of peritoneal dialysis-related peritonitis. Perit Dial Int. 23(6):580-586

7. Xiangmei Chen (2010) Peritoneal dialysis standard operating procedure (SOP). People's Military medical press, 1st edn. Beijing, China

8. Li PK, Szeto CC, Piraino B, de Arteaga J, Fan S, Figueiredo AE, Fish DN, Goffin E, Kim YL, Salzer W, Struijk DG, Teitelbaum I, Johnson DW (2016) ISPD peritonitis recommendations: 2016 update on prevention and treatment. Perit Dial Int 36(5):481-508. https://doi.org/10.3747/pdi.2016.00078

9. Boyce JM, Pittet D on behalf of Healthcare Infection Control Practices Advisory Committee; HICPAC/SHEA/ APIC/IDSA Hand Hygiene Task Force. Guideline for Hand Hygiene in Health-Care Settings. Recommendations of the Healthcare Infection Control Practices Advisory Commit- tee and the HICPAC/SHEA/ APIC/IDSA Hand Hygiene Task Force. Society for Healthcare Epidemiology of America/ Association for Professionals 
in Infection Control/Infectious Diseases Society of America. MMWR Recomm Rep 2002; 51:1-45,CE1-4.

10 Szeto C-C, Li PK-T (2019) Peritoneal dialysis-associated peritonitis. Clin J Am Soc Nephrol. 14(7):1100-1105

11. Dong J, Chen Y (2010) Impact of the bag exchange procedure on risk of peritonitis. Perit Dial Int 30:440-447

12. Russo R, Manili L, Tiraboschi G, Amar K, De Luca M, Alberghini E et al (2006) Patient re-training in peritoneal dialysis: why and when it is needed. Kidney Int Suppl 103:S127-S132

13. Piraino B, Bernardini J, Brown E, Figueiredo A, Johnson DW, Lye WC, Price V, Ramalakshmi S, Szeto CC (2011) ISPD position statement on reducing the risks of peritoneal dialysis-related infections. Perit Dial Int. 31(6):614-630. https://doi.org/10.3747/ pdi.2011.00057

14 Cho Y, Johnson DW (2014) Peritoneal dialysis-related peritonitis: towards improving evidence, practices, and outcomes. Am J Kidney Dis 64(2):278-289. https://doi.org/10.1053/j.ajkd.2014. 02.025

15 Firanek C, Guest S (2011) Hand hygiene in peritoneal dialysis. Perit Dial Int 31:399-408. https://doi.org/10.3747/pdi.2010.00239

16. Ye Y, Zhang X, Liu Y, Lou H, Shou Z (2017) Research on handwashing techniques of peritoneal dialysis patients From Yiwu, Southeast China. Ther Apher Dial 21(2):200-205. https://doi.org/ 10.1111/1744-9987.12514

17. Perl J, Fuller DS, Bieber BA, Boudville N, Kanjanabuch T, Ito Y, Nessim SJ, Piraino BM, Pisoni RL, Robinson BM, Schaubel DE
(2020) Peritoneal dialysis-related infection rates and outcomes: results from the peritoneal dialysis outcomes and practice patterns study (PDOPPS). Am J Kidney Dis 76(1):42-53. https://doi.org/ 10.1053/j.ajkd.2019.09.016

18. Boudville N, Johnson DW, Zhao J, Bieber BA, Pisoni RL, Piraino B, Bernardini J, Nessim SJ, Ito Y, Woodrow G, Brown F, Collins J, Kanjanabuch T, Szeto CC, Perl J (2019) Regional variation in the treatment and prevention of peritoneal dialysis-related infections in the Peritoneal Dialysis Outcomes and Practice Patterns Study. Nephrol Dial Transplant 34(12):2118-2126. https://doi.org/ 10.1093/ndt/gfy204

19. Zelenitsky SA, Howarth J, Lagacé-Wiens P, Sathianathan C, Ariano R, Davis C, Verrelli M (2017) Microbiological trends and antimicrobial resistance in peritoneal dialysis-related peritonitis 2005 TO 2014. Perit Dial Int. 37(2):170-176. https://doi.org/10. 3747/pdi.2016.00136

20. Kerschbaum J, Konig P, Rudnicki M (2012) Risk factors associated with Peritoneal-dialysis-related peritonitis. Int J Nephrol. 2012:483250

Publisher's Note Springer Nature remains neutral with regard to jurisdictional claims in published maps and institutional affiliations. 